\title{
Real-time prescription monitoring: lessons from Tasmania
}

\begin{abstract}
Peter Boyles
Chief Pharmacist,

Pharmaceutical Services

Branch, Public Health

Services, Department of

Health and Human Services, Hobart
\end{abstract}

\section{Keywords}

opioids, pain, prescription drug monitoring programs

Aust Prescr 2019;42:48-9

https://doi.org/10.18773/ austprescr.2019.007
Real-time prescription monitoring programs are effective in improving clinical decision-making. They also reduce doctor shopping and the diversion of controlled substances.' These programs therefore have the potential to reduce harm from prescription drugs including deaths from opioid poisoning. Coroners, and pharmacy and medical representative groups have been calling for real-time prescription monitoring for many years.

In 2009, Tasmania was the first state to implement a real-time prescription monitoring system called DORA (Drugs and Poisons Information System Online Remote Access). Initially, DORA only provided information to Tasmanian Department of Health regulators but had plans to implement access for clinicians as a priority. From 2011, DORA enabled clinicians to view clinical information and dispensing data about Schedule 8 drugs, and current and past authorities issued for the prescribing of Schedule 8 drugs to specific patients. It can confirm whether or not the patient has been declared drug-dependent or a drug seeker (as defined by the Tasmanian Poisons Act 1971) and if the patient has ever been treated in Tasmania for an opioid substance use disorder. Currently the use of DORA by clinicians is not mandatory, however this is a requirement of many US systems and could become mandatory in some Australian jurisdictions.

Victoria started a trial of its monitoring in 2018, and other jurisdictions such as Western Australia and the Australian Capital Territory are expected to follow. The Australian Government is supporting states and territories to adopt real-time prescription monitoring as soon as possible. It has been actively facilitating the development of policies and software solutions to enable access to patient-specific information across state and territory borders.

Victorian Coroners' data suggest approximately $85 \%$ of deaths from pharmaceutical poisoning are from medicines legally prescribed and dispensed to the deceased. Very few deaths are due to diverted prescribed medicines. Only about $25 \%$ of all deaths from prescription medicine poisoning involved multiple prescribers. ${ }^{2}$

Between 2012 and 2016, 3993 Australians died from prescription opioid poisoning. These figures represent an increase of approximately $113 \%$ compared with 2002-06 (1877 opioid poisoning deaths). Tasmania's per capita death rate from prescription opioids was approximately $30 \%$ above the national average between 2002 and 2006 and changed to approximately $27 \%$ below the national average between 2012 and 2016 according to Australia's Annual Overdose Report 2018. ${ }^{3}$ It is important to note that Tasmanian Government records show that the number of patients prescribed Schedule 8 opioids for persistent non-malignant pain increased from 1600 (2002) to 6400 (2016). While the Australian figures represent an unacceptable increase in preventable deaths, there has been a significant reduction in individual patient risk in Tasmania.

It should not be assumed this change was due solely to the implementation of DORA. In reality, the changes seen in Tasmania regarding these preventable deaths is more likely to have occurred following a significant increase in the rigour, sophistication and subsequent effectiveness of expert clinician-led, clinical governance regulatory activities and improvements in the awareness of GPs and pharmacists. These have been possible due to the efforts of local pain specialists, addiction specialists, pharmacists and GPs collaboratively providing advice to coordinate support to the clinical-regulatory pharmacists of the Tasmanian Government's Pharmaceutical Services Branch. Through these collective efforts, Tasmania has developed a robust, evidence-based, qualityassurance system that assists clinicians to safely and effectively treat patients via patient-and-clinicianspecific audit-and-feedback mechanisms. ${ }^{4}$

DORA and the policies and procedures of the Pharmaceutical Services Branch have been the subject of as yet unpublished reviews conducted by Public Health Services Medical Registrars. Conclusions included 'DORA is contributing to a reduction in opioid-related harms in Tasmania, but the extent of the reduction could not be determined'.

Serious consideration needs to be given to all the options available to us to collectively tackle the injudicious prescribing of opioids. This is most often by a single medical practitioner or medical practice which time and again leads to what Tasmanian Coroner Carey in 2016 referred to as 'likely and avoidable' deaths. ${ }^{5}$ The time has come to refuse to accept these poisoning deaths as inevitable 


\section{<ustralian Prescriber}

VOLUME 42 : NUMBER 2 : APRIL 2019

'accidents', but rather acknowledge our collective failure to provide safe care.

Adoption of real-time monitoring throughout Australia might well avoid the high mortality from prescription opioid overdose currently reported in the USA. The Tasmanian clinical governance regulatory model of risk-based, targeted individual clinician accountability has improved standards of care in the clinical management of patients with chronic non-cancer pain. A persistent and consistent message has been communicated to clinicians about the importance of safe and appropriate treatment of patients with chronic non-cancer pain. This appears to be having an impact on clinical practice, for example the average prescribed oral morphine equivalent daily dose declined in Tasmania from $97.2 \mathrm{mg}$ in 2004 to $35.1 \mathrm{mg}$ in 2017. It is our collective challenge to maintain and build on these evidence-guided improvements in clinical practice.

DORA has certainly proved to be a valuable tool for many clinicians. However, it is the partnership of expert clinicians in general practice, pain, and addiction medicine, working constructively with community and Tasmanian Government pharmacists, that has driven the change in the environment and clinical practice. This has resulted in better outcomes for the Tasmanian community and this partnership should be seriously considered by other Australian jurisdictions as part of their implementation of realtime monitoring. $<$

\section{Conflict of interest: none declared}

\section{REFERENCES}

1. Prescription Drug Monitoring Program Center of Excellence at Brandeis University. Briefing on PDMP Effectiveness. Updated Sep 2014. http://www.pdmpassist.org/content/ pdmp-evaluations [cited 2019 Mar 1]

2. Turning Point, Eastern Health [Internet]. Dwyer J, presenter. Talking point: New insights into Victorian pharmaceutical drug overdose death. Talking Point Alcohol and Other Drug Seminar Series. 2017 Feb 22. www.turningpoint.org.au/ education/talking-point/victorian-pharmaceutical-drugoverdose-death [cited 2019 Mar 1]

3. Penington Institute. Australia's annual overdose report 2018. Melbourne: Pennington Institute; 2018

http://www.penington.org.au/australias-annual-overdosereport-2018 [cited 2019 Mar 1]
4. Pharmaceutical Services Branch, Tasmanian Government Process for assessing and granting authorities to prescribe Schedule 8 drugs under Section 59E of the Tasmanian Poisons Act 1971. https://www.dhhs.tas.gov.au/psbtas [cited 2019 Mar 1]

5. Carey S, Coroner for Barnes, DJ. Record of investigation into death (without inquest). 2016 May 25. Magistrates Court of Tasmania, Coronial Division. https://www.magistratescourt.tas.gov.au/about_us/ coroners/coronial_findings [cited 2019 Mar 1] 